\title{
Desarrollo e Instrumentación de un Proceso de Vigilancia Tecnológica basado en Protocolos de Revisión Sistemática de la Literatura
}

Ana López ${ }^{(1)}$, Diego Méndez ${ }^{(1)}$, Andrés $\mathrm{Paz}^{(2)}$ y Hugo Arboleda ${ }^{(1) *}$

(1) Universidad Icesi, Calle 1 No. 122 - 135, Cali, Colombia. (e-mail: ana.lopez@correo.icesi.edu.co, diego.mendez@correo.icesi.edu.co; hfarboleda@icesi.edu.co)

(2) Université du Québec, École de Technologie Supérieure, Montréal, Canada

(e-mail: andres.paz@me.com)

* Autor a quien debe ser dirigida la correspondencia.

Recibido Dic. 10, 2015; Aceptado Feb. 15, 2016; Versión final Mar. 2, 2016, Publicado Ago. 2016

\section{Resumen}

Este artículo presenta un proceso de vigilancia tecnológica que considera las actividades asociadas al proceso de realizar revisiones sistemáticas de literatura. La vigilancia tecnológica forma parte del sistema de gestión de innovación e investigación en contextos industriales. La propuesta tiene como objetivo el brindar acceso, gestión y contextualización de conocimientos científicos y técnicos que están a la vanguardia en tecnologías de soporte a la estrategia del negocio. Las revisiones sistemáticas de la literatura consideran un proceso detallado para estudiar los avances en un campo particular del conocimiento. Este tipo de revisiones constituye un facilitador de procesos efectivos de vigilancia tecnológica. El carácter innovador del proceso que se presenta es bidimensional. Por una parte se integran buenas prácticas para realizar vigilancia tecnológica y revisiones sistemáticas, y por otra se propone una herramienta de soporte (LOXO) para la ejecución de procesos de vigilancia tecnológica.

Palabras clave: vigilancia tecnológica; revisiones sistemáticas de literatura; buenas prácticas; herramienta de soporte; contextos industriales

\section{Development and Implementation of a Technology Surveillance Process Based on Systematic Literature Review Protocols}

\begin{abstract}
This paper presents a technology surveillance process that takes into account activities associated with the process of conducting systematic literature reviews. Technology surveillance is a part of the research and innovation management system in industrial contexts. The aim of the proposal is to provide access, management and contextualization of scientific and technical knowledge to be at the forefront of technologies for supporting business strategy. Systematic literature reviews consider a detailed process to study the state of the art in a particular field of knowledge. This type of reviews constitutes a facilitator of effective technology surveillance processes. The innovative nature of the process is two-fold. On the one hand, integrates best practices to carry out technology forecasting and systematic literature reviews and on the other, a support tool (LOXO) is proposed for the execution of technology surveillance processes.
\end{abstract}

Keywords: technology surveillance; systematic literature reviews; best practices; support tool; industrial contexts 


\section{INTRODUCCIÓN}

Organizaciones en todos los sectores son llamadas a asumir una actitud proactiva en relación a la adquisición de soluciones tecnológicas que sean la base de su estrategia organizacional. La Vigilancia Tecnológica (VT) se define como un proceso sistemático de investigación que permite captar información sobre tecnologías de información ( $\mathrm{TI}$ ) y convertir esta información en conocimiento para apoyar la toma de decisiones (UNE 166006:2011, 2011). La VT hace parte del sistema de gestión de investigación, desarrollo e innovación, y brinda acceso, gestión y contextualización de los conocimientos científicos y técnicos que permitirán suministrar soluciones a la medida a partir de información individualizada (Johannessen y Olsen, 2010). Un proceso de VT utiliza varios enfoques de investigación existente para recolectar la información. Entre ellos se encuentra la revisión de publicaciones técnicas en bases de datos. Actualmente la norma UNE 166006 de 2011, establecida internacionalmente por AENOR la cual es miembro de la organización ISO (organización internacional para la estandarización), enuncia los conceptos que enmarcan la VT y plantea las actividades que conforman el proceso. La descripción de procesos presentada por la norma es de alto nivel, omitiendo detalles procedimentales de implementación.

Kitchenham (2004) basándose en buenas prácticas de investigación en áreas de biología y medicina, definió un procedimiento para llevar a cabo Revisiones Sistemáticas de Literatura (RSL) en el área de la ingeniería de software. Este trabajo de Kitchenham es el trabajo seminal de las RSL y propuestas de mejoramiento del proceso de realización de las RSL en el área de la ingeniería de software. Las RSL permiten, entre otras actividades, recopilar las publicaciones técnicas en una temática específica. Desde entonces, diferentes autores han abordado el tema analizando las problemáticas que se tienen para obtener información de calidad (Staples y Niazi, 2007) planteando mejoras para obtener dicha información (Kitchenham et al., 2010), investigando cómo mejorar el proceso de búsqueda (Dieste y Padua, 2007; Skoglund y Runeson, 2009) y presentando nuevas propuestas que sugieran un procesos ágil (Abdala, 2014; Biolchini et al., 2005; Kitchenham y Brereton, 2013; Zhuoqun et al., 2013).

La vigilancia tecnológica (VT) es una práctica de observación periódica, organizada y selectiva del entorno tecnológico que tiene por objetivo la búsqueda, selección y análisis de información sobre tecnología para convertirla en conocimiento que apoyará la toma de decisiones. El sistema de VT abarca (i) las políticas de vigilancia definidas por las compañías a partir de los objetivos que se esperan obtener de la implementación de la VT, (ii) el proceso de investigación, y (iii) las decisiones que se tomen a partir de los resultados que arroje el sistema (UNE 166006:2011, 2011). Las acciones derivadas son formuladas en torno a una innovación, una propuesta de mejora, la anticipación de una situación, o el aprovechamiento de oportunidades que pasarán a ser parte de proyectos en otras áreas de la organización.

El proceso de investigación en el sistema de la VT (ver Fig. 1-a) comprende los subprocesos de (1) identificación, que incluye actividades de identificación de necesidades de información, fuentes internas y externas de información, y planificación de la realización de la VT; (2) planificación, donde se dimensionan y planifican los recursos requeridos para realizar la VT; (3) búsqueda, tratamiento y validación de la información, a partir de una estrategia definida donde se indica terminología, palabras claves y operadores a utilizar según la fuente de información; (4) puesta en valor, que incluye la integración de datos para conseguir sinergia, interpretación de la información en pro de realizar pronósticos y posible evolución de un tema; (5) distribución de la información, asociada con los involucrados en el producto de la realización de la VT; y (6) recopilación de resultados.

Las revisiones sistemáticas de literatura (RSL) permiten hacer una evaluación e interpretación de la documentación disponible para una temática en particular, mediante la aplicación de un proceso fiable, riguroso, auditable y repetible (Kitchenham, 2004). Las fuentes de investigación corresponden a publicaciones técnicas, lo que incluye bibliotecas digitales, revistas especializadas, reportes técnicos, memorias de congresos y trabajos en progreso (Kitchenham y Charters, 2007). El protocolo está compuesto por 13 actividades divididas en las etapas de planeación, ejecución y reportes, según el objetivo de cada una. En la primera etapa, planeación, se identifican las necesidades u objetivos de investigación y se plantea la estrategia de búsqueda para conseguir los mismos. En la etapa de ejecución se lleva a cabo el plan definido y se consiguen los artículos obtenidos en la consulta para ser clasificados, analizados y sintetizados en un resumen. En la última etapa, reportes, se elabora el informe final de la investigación y se da a conocer a los interesados los resultados del proceso. La Fig. 1-b presenta el proceso propuesto por Kitchenham, en donde se propone que luego de desarrollar una búsqueda inicial y hacer una primera lectura de los documentos obtenidos, y ante la evidencia de existencia de otros términos para identificar los artículos objetivo, se ajustan los criterios de inclusión y/o exclusión. En la actividad Definir Estrategia se plantea la estrategia de búsqueda y valoración de datos que se utilizará en el proceso, por lo que allí se definen las cadenas de búsqueda y los criterios de calidad que permitirán clasificar y puntuar los artículos encontrados. La actividad Desarrollar Búsqueda indica la realización de la búsqueda con la respectiva 
documentación de la actividad realizada, para que de esta forma se considere pueda ser sistemática y repetible por otros investigadores quienes deberían obtener resultados similares al ejecutar la misma estrategia. En la actividad Sintetizar Datos se detalla la forma de analizar la información obtenida, para así procesarla y dar un balance de lo obtenido en la consulta de acuerdo a los objetivos de investigación planteados.

Este artículo presenta un proceso de VT que cubre el conjunto de actividades requeridas para realizar revisión de publicaciones técnicas en bases de datos. El carácter innovador del proceso que se presenta es bidimensional. Por una parte se integran buenas prácticas para realizar las RSL, adaptando particularmente el proceso presentado por Kitchenham (2007) al contexto de VT. Por otra parte, se instrumenta el proceso mediante la especificación e implementación de una herramienta de soporte.

\section{VIGILANCIA TECNOLÓGICA Y PROTOCOLO RSL}

El proceso de investigación para realizar la VT y el protocolo para realizar las RSL son procesos organizados de búsqueda que tienen como objetivo presentar un análisis sobre un tema objetivo para tomar decisiones sobre el futuro de una labor. El primero está dirigido hacia la industria y hace parte de un amplio marco en donde la dirección estratégica de la organización es el principal eslabón del proceso, tanto por la política de vigilancia como por las decisiones que se toman a partir de los resultados de la VT, lo que impacta directamente la gestión de innovación y la gestión del conocimiento. Sin embargo, los pasos para ejecutar VT son guías generales en donde se indica la actividad a realizar pero no se menciona como debe realizarse.

Las RSL persiguen objetivos similares a los de la VT, aunque su enfoque es más de tipo investigativo por lo que son ampliamente utilizadas en la academia, donde es de interés constante conocer si un tema ha sido explorado por otros autores y cuál ha sido su enfoque para así determinar cuáles podrían ser los futuros trabajos en ese campo. La estructura de una RSL puede complementar lo determinado actualmente para el proceso de investigación de VT, definiendo cómo realizar una búsqueda sistemática que permita asegurar la calidad de los resultados obtenidos y que pueda repetirse si posteriormente se requiere reevaluar los requisitos que la generaron. Además, la ejecución del proceso define el detalle de cada actividad y usa una metodología que ha madurado con rapidez por su gran acogida en el mundo investigativo de las tecnologías de información.

La Fig. 1 presenta las relaciones existentes entre los procesos de VT y RSL. Dichas relaciones toman como referencia lo que en la VT corresponde al enfoque relacionado con búsqueda en bases de datos (publicaciones técnicas), ya que éste es el campo de acción de las RSL. Ambos procesos presentan 3 etapas para desarrollar sus actividades: Planeación, Ejecución y Reportes. Como se presenta en la figura, en lo que se refiere a la etapa de planeación, tanto la VT como las RSL contemplan actividades para definir la necesidad que se tiene y la estrategia que se usará para conseguir la información requerida. En lo que correspondería a la etapa de ejecución, en ambos casos se realizan actividades de búsqueda y de análisis y valoración de información para posteriormente realizar una síntesis de los resultados. En la parte de reportes, se comparte la actividad de informar sobre los resultados del proceso.

\section{PROCESO PARA VT DE PUBLICACIONES TÉCNICAS}

El proceso propuesto en esta sección considera los elementos de base del proceso para VT UNE 166006:2011, 2011) y el protocolo de RSL propuesto por Kitchenham y Brereton (2013). Se destaca una etapa ágil de planeación, enfocando los esfuerzos de los interesados en la definición de los objetivos y los criterios de la búsqueda. La Fig. 2 presenta el proceso con el detalle de las actividades que lo componen. En la etapa de Planeación, la actividad de Identificar Necesidad pretende definir la meta específica del proceso búsqueda y lo que se espera conseguir con la misma. Incluye la redacción de objetivos. Definir Estrategia considera el establecimiento de la estrategia, que consiste en la definición de las cadenas búsqueda a utilizar para obtener la información y artículos necesarios en los motores de búsqueda. Las cadenas de búsqueda se componen de cadenas de dominio, criterios de filtrado positivo y negativo, criterios de exclusión, y criterios de calidad. Las cadenas de dominio son las cadenas que se requieren para realizar la búsqueda de títulos en los motores de búsqueda. Se originan a partir de los objetivos de la búsqueda dentro de la definición de la necesidad. Es importante destacar que los dominios son considerados criterios de filtrado positivo y se separan para efectos de realización de búsquedas. Los criterios de filtrado positivo son utilizados para realizar las búsquedas por títulos en los motores de búsqueda. Los criterios de filtrado negativo son cadenas que serán utilizadas para excluir de los artículos obtenidos aquellos que en su resumen contengan alguna de las cadenas descritas. Los criterios de exclusión son exclusiones a partir de criterios más complejos que los de filtrado negativo, y por ello se requiere de una lectura e interpretación del resumen y/o introducción de los trabajos por parte del revisor para definir su exclusión o no. 


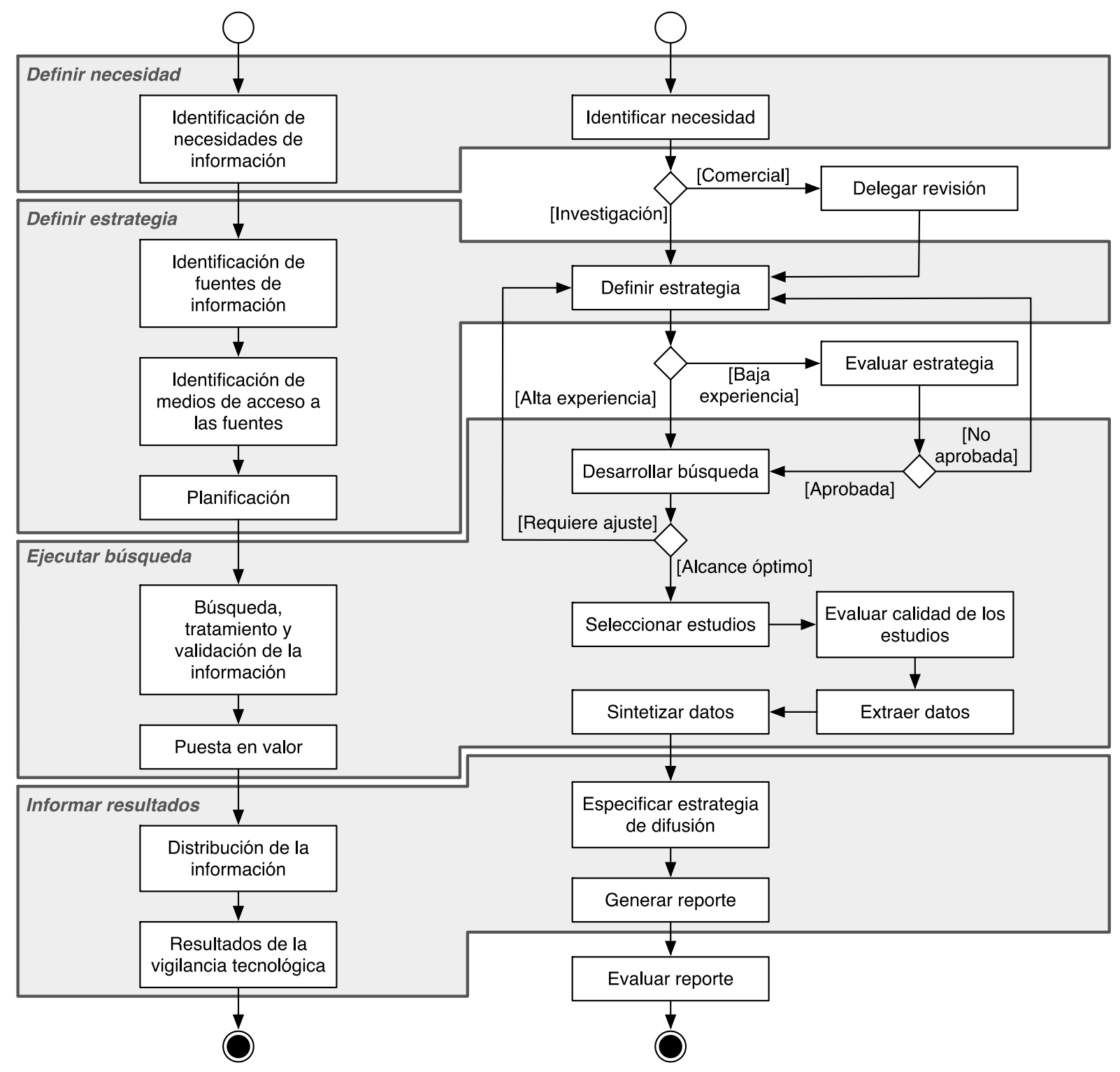

Fig. 1: (a) El proceso de VT (izquierda) y (b) el protocolo de RSL (derecha)

En la etapa de Ejecución, la actividad Desarrollar Búsqueda consiste en ejecutar la búsqueda y obtener los resultados. Se ingresan las cadenas de dominio, y los criterios de filtrado positivo y negativo. Durante Seleccionar Estudios se realiza una primera lectura de los artículos obtenidos y se aplican los criterios de exclusión manuales para pasar a Evaluar Calidad De Los Estudios, que consiste en aplicar los criterios de calidad definidos en la actividad de Establecer Estrategia. Extraer Datos consiste en definir las variables a ser tabuladas y que se requieren para poder dar respuesta al objetivo de la VT. Una vez se tienen claras estas variables, se hace necesario realizar una lectura sobre los artículos obtenidos para proceder a poder tabular correctamente cada estudio con las variables analizadas. Durante la actividad de Sintetizar Datos se toman las variables tabuladas por cada artículo y se generan informes y gráficos que resuman los resultados obtenidos. En este punto es necesario realizar una validación de lo definido en la actividad de Identificar Necesidad, para asegurar que los gráficos e informes a presentar estén alineados con la necesidad identificada inicialmente por la organización. Finalmente, en la etapa de Divulgación, durante la actividad de Comunicar Resultados a los Interesados se socializa el proceso y los resultados obtenidos.

\section{HERRAMIENTA DE SOPORTE AL PROCESO DE VT}

Para la aplicación del protocolo de VT propuesto es necesario contar con herramientas que permitan automatizar tareas de búsqueda y contar con opciones donde los usuarios puedan llevar información sobre el estado de la VT, controlar los artículos leídos, poder hacer control de calidad de los mismos y generar gráficas que sinteticen los resultados obtenidos. Esta sección presenta elementos destacados de una especificación de referencia, incluyendo retos que debe asumir la implementación de la herramienta, el alcance funcional, las restricciones, y los escenarios de calidad. 


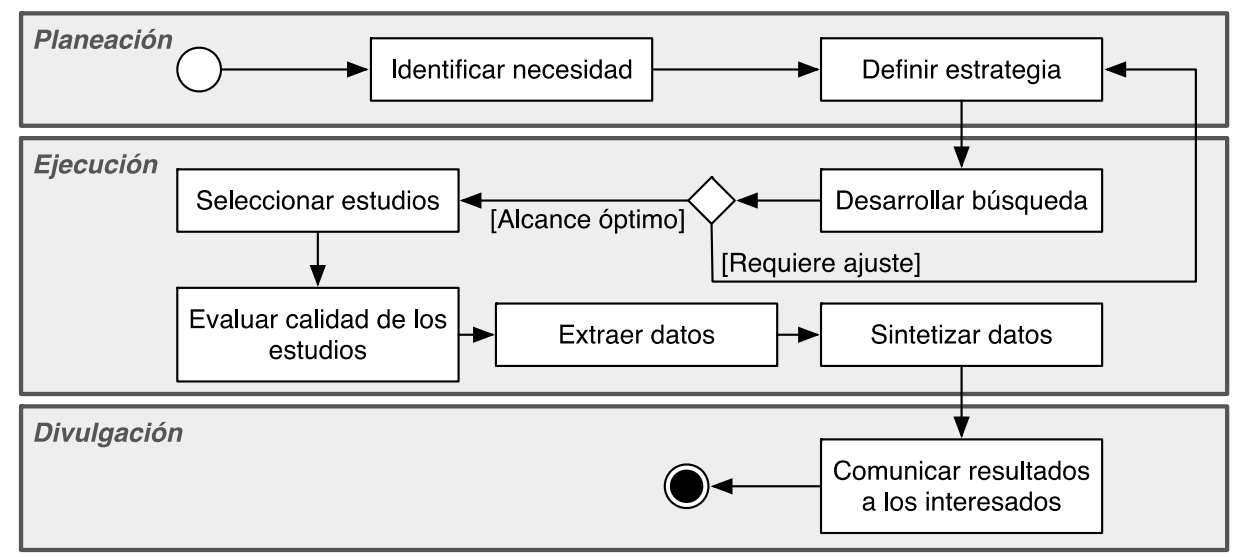

Fig. 2: Proceso para VT propuesto

\section{Retos y alcance funcional}

Entre los retos que aborda un proyecto de desarrollo de una herramienta de soporte para el desarrollo del proceso de VT propuesto en la Sección 4 se destacan (1) minimizar tiempos de investigación en la industria, (2) aumentar el porcentaje de cumplimiento de proyectos, (3) disminuir el tiempo de evaluación de proveedores y (4) disminuir el número de proyectos mal concesionados aumentando la efectividad en la asignación. Estos retos, acompañados de métricas y umbrales, se convierten en motivadores del negocio que deben ser medidos en términos del retorno de inversión del proyecto de desarrollo; dicho retorno de inversión se puede ver reflejado en indicadores económicos directos, posicionamiento en el mercado, clima organizacional, y ventaja competitiva por innovación entre otros. Para dar cobertura a la aplicación del proceso se requiere implementar un conjunto de requerimientos funcionales. La Tabla 1 presenta un conjunto destacado de dichos requerimientos asociados a actividades del proceso.

\section{Restricciones y escenarios de calidad}

En términos de restricciones, los motores de búsqueda que la herramienta debe utilizar deben incluir, al menos, ACM, IEEE, SpringerLink y Science Direct. Esto con el fin de asegurar la cantidad y calidad de los artículos a retornar. La selección de los anteriores motores de búsqueda se debe a que son los ofrecidos por las editoriales más representativas de trabajos de investigación sobre tecnología. Además, son las fuentes bibliográficas recurrentes en los estudios sistemáticos de literatura en dicho campo (Abdala, 2014; Dias de Carvalho et al., 2014; Kitchenham y Brereton, 2013; Petersen et al., 2008; Yang et al., 2014). Los escenarios de calidad son una estrategia ampliamente utilizada para representar requerimientos no funcionales en los sistemas de software en términos de sus atributos de calidad (Bass et al., 2012). La especificación de un escenario de calidad se realiza de forma independiente de toda funcionalidad del sistema y describe solo cualidades esperadas en él por parte de las personas interesadas. En relación a escenarios de calidad, se establecen como mínimo la interoperabilidad y usabilidad en diferentes navegadores web, la modificabilidad de la aplicación soportada por un deseable desarrollo por capas y despliegue sobre un servidor de aplicaciones Java como Tomcat.

\section{Diseño e implementación de una herramienta de soporte}

Loxo es una herramienta que tiene por objetivo ser el soporte para la realización de una vigilancia tecnológica, la cual ha sido diseñada para aplicar el proceso de VT propuesto en la sección anterior. Loxo fue implementada basándose en un sistema legado que realiza consultas en los motores de búsqueda IEEEXplore, ACM, SpringerLink y Science Direct, desarrollado como parte de un trabajo de fin de maestría de la Universidad Icesi de Cali, Colombia (Abdala, 2014). La herramienta se encuentra disponible para su descarga en https://github.com/unicesi/loxo/releases. Entre los requerimientos implementados, tomados de la especificación de referencia, el sistema permite ingresar e importar las cadenas de búsqueda (cadenas de dominio, y cadenas de filtrado positivo y negativo), realizar búsqueda en los motores soportando expresiones regulares, y generar un archivo BIBTeX consolidado que incluye el resultado final de la búsqueda para ser usado en la herramienta Mendeley.

Marshall y Brereton (2013) realizaron un estudio para identificar y clasificar herramientas cuyo objetivo sea la automatización parcial o total del proceso de realización de las RSL. Los autores del estudio resaltan un crecimiento en el número de propuestas. Loxo se suma a este conjunto de herramientas, y de acuerdo a la clasificación expuesta en dicho estudio, Loxo busca soportar todo el proceso de realización de una RSL. Sin embargo, el carácter innovador de Loxo es que está dirigida a soportar procesos de VT mediante la incorporación de buenas prácticas para realizar las RSL. 


\section{VALIDACIÓN Y RESULTADOS}

La validación del proceso propuesto y su instrumentación se realizó en el contexto de un proyecto de VT ejecutado en una organización dedicada al bienestar social de trabajadores y beneficiarios afiliados. Esta organización brinda servicios subsidiados de educación, vivienda, recreación y responsabilidad social para que la población de menos recursos pueda acceder a estos servicios a un bajo costo. Para lograr sus objetivos y poder prestar sus servicios adecuadamente, la organización se apalanca en el uso de diversas soluciones tecnológicas que le permitan contar con la información de los afiliados y sus derechos en línea. La organización hizo uso del proceso y la herramienta aquí propuestos en su interés de realizar vigilancia tecnológica en el contexto de estrategias para implementar una línea de procesos de desarrollo de software (SPrL, por sus siglas en inglés). Posteriormente, los autores de este trabajo compararon resultados del proceso con los resultados de una RSL realizada acerca del tema de SPrL por Dias de Carvalho et al. (2014). El ejercicio de comparación permitió conocer la asertividad del proceso y la herramienta, midiéndose contra un proceso de RSL que no incluye principios de VT y que fue desarrollado manualmente.

Tabla 1: Extracto de requerimientos funcionales para una herramienta de soporte al proceso VT

\begin{tabular}{|l|}
\hline \multicolumn{1}{|c|}{ Actividad Desarrollar Búsqueda } \\
\hline RF01: Permitir ingresar e importar las cadenas de búsqueda (cadenas de dominio, y cadenas de filtrado positivo y \\
negativo). \\
RF02: Contar con un mecanismo que permita importar los resultados de búsquedas ejecutadas de forma manual. \\
RF03: Recibir una expresión regular como cadena de búsqueda que integre cadenas dominio, y cadenas de filtrado \\
positivo y negativo. \\
RF04: Exportar a un archivo basado en caracteres, la traza de pasos ejecutados por la herramienta en el proceso \\
de búsqueda. \\
RF05: Realizar búsqueda en los motores de búsqueda. \\
RF06: Generar un archivo BIBTeX consolidado con el resultado final de la búsqueda para ser usado en una \\
herramienta de administración de referencias tal como Mendeley \\
\hline \multicolumn{1}{|c|}{ Actividad Seleccionar Estudios } \\
\hline RF07: Agrupar artículos para revisión por autores y asignarlos para lectura a los revisores. \\
RF08: Permitir la asignación de pares revisores. \\
RF09: Permitir cargar objetivos a cumplir con la aplicación del protocolo de VT propuesto. \\
RF10: Permitir cargar y personalizar criterios de calidad. \\
\hline \multicolumn{1}{|c|}{ Actividad Evaluar Calidad de los Estudios } \\
\hline RF11: Permitir registrar información sobre avance de revisiones y el aseguramiento de calidad. \\
\hline Actividad Sintetizar Datos \\
\hline RF12: Contar con un módulo de reportes y generación graficas e informes de síntesis. \\
\hline
\end{tabular}

\section{Ejecución del proceso de VT propuesto}

De manera ilustrativa, a continuación se presenta un subconjunto de las entradas, salidas y resultados relacionados en la aplicación del proceso propuesto. Los detalles se encuentran documentados en (López y Mendez, 2015). En relación al conjunto de objetivos de búsqueda, se definieron los siguientes: 1) caracterizar los enfoques desde los que se ha abordado SPrL; 2) identificar los elementos del proceso de software que han sido objetivo de variabilidad; 3) establecer los lenguajes de modelado que se usan para SPrL; 4) reseñar los modelos y estándares de calidad que se han utilizado en SPrL; y 5) conocer cómo se están validando los trabajos de SPrL y si existen herramientas que los apoyen. Un subconjunto de las cadenas de búsqueda que se utilizaron para la aplicación del proceso es presentado en la Tabla 2.

Los criterios de exclusión fueron los siguientes: i) publicaciones que correspondan a procedimientos, reportes técnicos, tutoriales o cualquier trabajo no evaluado por pares en comités reconocidos; ii) trabajos no relacionados a Ingeniería de Software; iii) el trabajo considera procesos de software sin tener un modelo de variabilidad; iv) el trabajo considera variabilidad en cuanto a comparación genérica, similitud estructural, minería de procesos, control estadístico de procesos, desviación de procesos o imprevisibilidad del proceso; y v) trabajos publicados fuera del rango de fechas comprendidas entre los años 1996 y el año 2014. Los motores seleccionados para la búsqueda fueron IEEEXplore, ScienceDirect y SpringerLink. Desarrollando el ejercicio de filtrado negativo manual, basado en la lectura de resúmenes de los trabajos encontrados, fueron excluidos 35 artículos de 48 retornados por Loxo, 29 de los cuales no estaban relacionados con ingeniería de software, 5 de ellos no tenían un modelo variabilidad y un artículo duplicado. Los artículos no excluidos fueron entonces revisados para la aplicación de los criterios de calidad, los cuales fueron tomados del trabajo de Abdala (2014), y que tiene como base los conceptos de Petersen et al.(2008). 
Tabla 2: Cadenas de búsqueda

\begin{tabular}{|ll|ll|l|}
\hline Cadenas de dominio & & Filtrado positivo & & Filtrado negativo \\
\hline Process line & Process lines & Variability & Variabilities & Product lines \\
Process family & Process families & Variation & Variations & Product line \\
Variant rich process & Business process & Variants & Variant & SLR \\
Variant-rich process & Software process & Commonalities & Commonality & Systematic review \\
Business processes & Process model & Similarity & Similarities & SR \\
Software processes & Process reuse & Feature models & Feature model & \\
Process models & Process tailoring & Feature modeling & Feature-based & \\
SPrL & & & & \\
\hline
\end{tabular}

Las actividades de Evaluar Calidad de los Estudios, Extraer Datos y Sintetizar Datos fueron realizadas utilizando una lista de chequeo y gráficas dinámicas que complementan a Loxo y fueron diseñadas previamente por autores de este trabajo (Abdala, 2014). Dichas gráficas permiten categorizar los artículos de acuerdo con su tipo, y calificar su idoneidad en cuanto a objetivos y calidad.

La Fig. 3 muestra la cantidad de artículos obtenidos por cada motor de búsqueda y el aporte que está realizando cada uno de ellos. La Fig. 4 presenta la clasificación en la que aporta cada uno de los artículos obtenidos, y se presenta la información según el año de publicación de cada artículo. La Fig. 5 presenta la distribución en la que se encuentran los artículos obtenidos, según el tipo de artículo y la base de datos desde donde fue obtenido. La Fig. 6 presenta una distribución de los artículos con respecto a su afinidad o aporte con los objetivos propuestos para el estudio. Por último se realizó una calificación a cada uno de los artículos para conocer su calidad y efectividad con respecto a los criterios de calidad y al propósito de la VT. La Fig. 7 presenta esta calificación y la cantidad de artículos por cada puntaje.

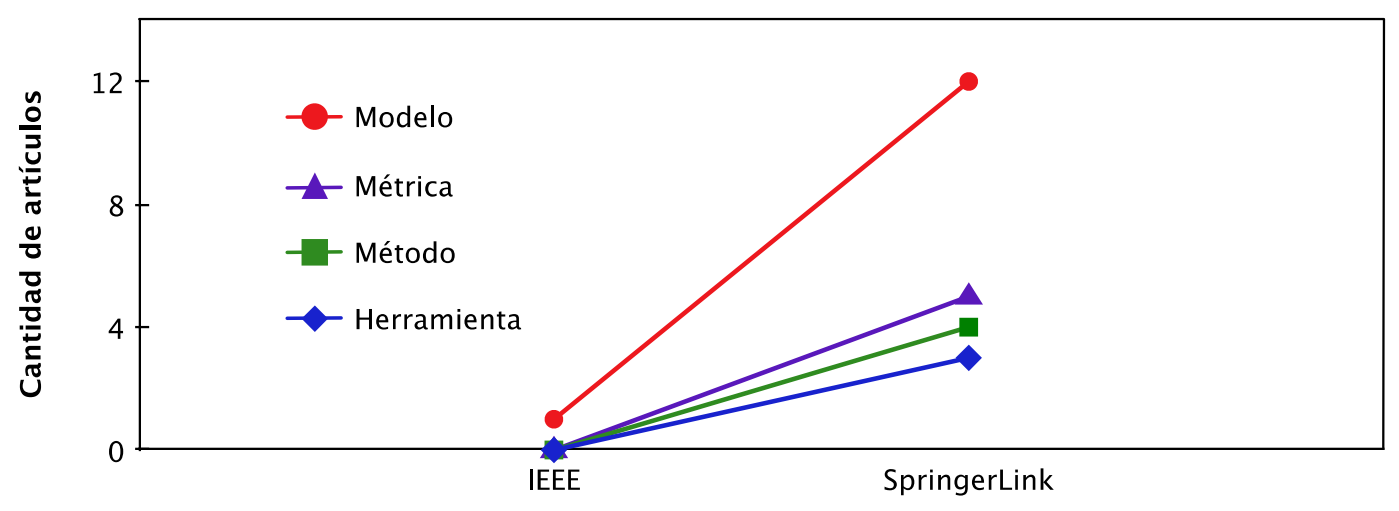

Bases de datos

Fig. 3: Aporte de artículos por base de datos

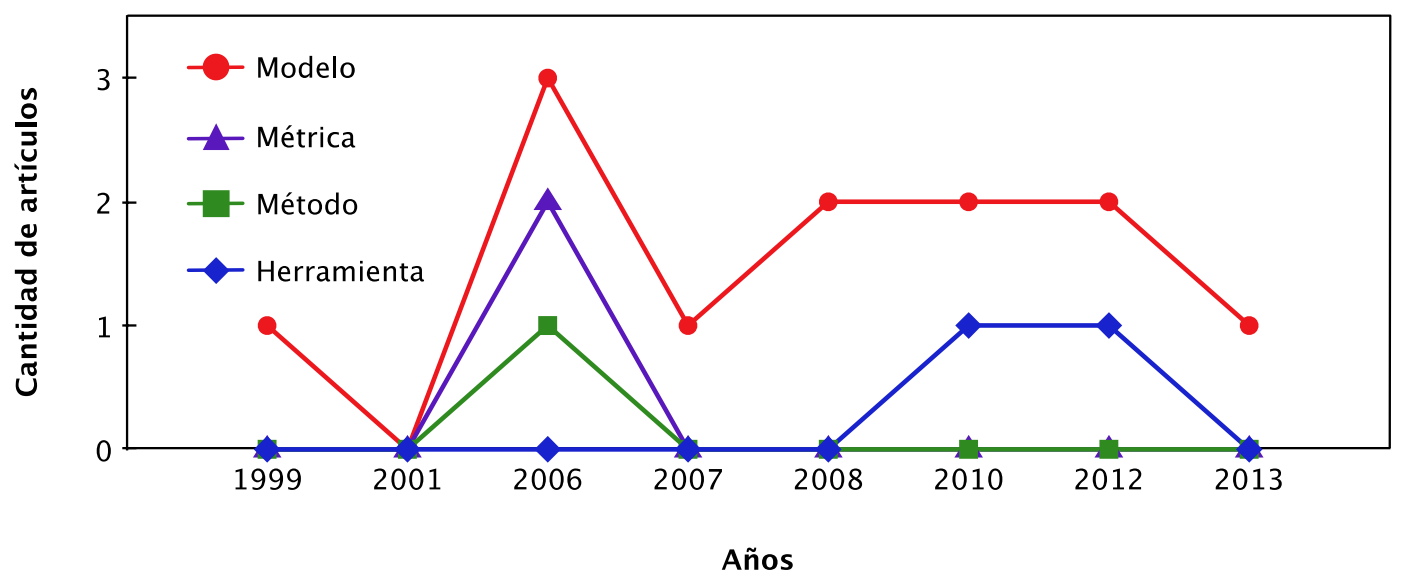

Fig. 4: Aporte de artículos por año 


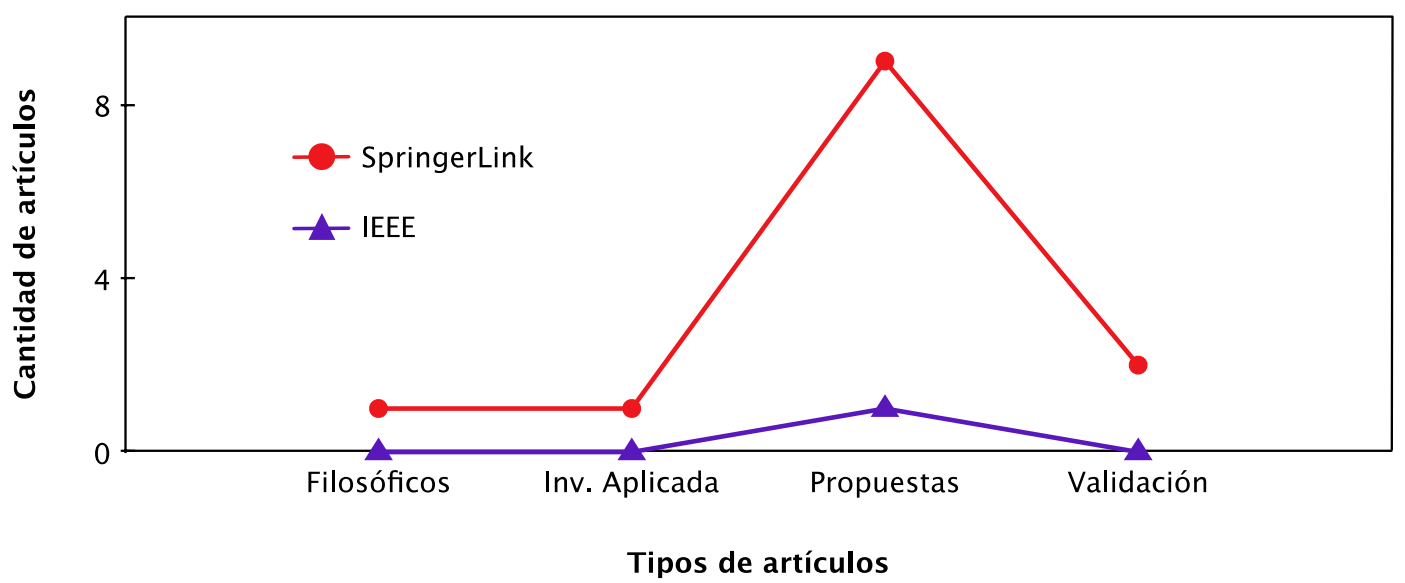

Fig. 5: Tipo de artículo por base de datos

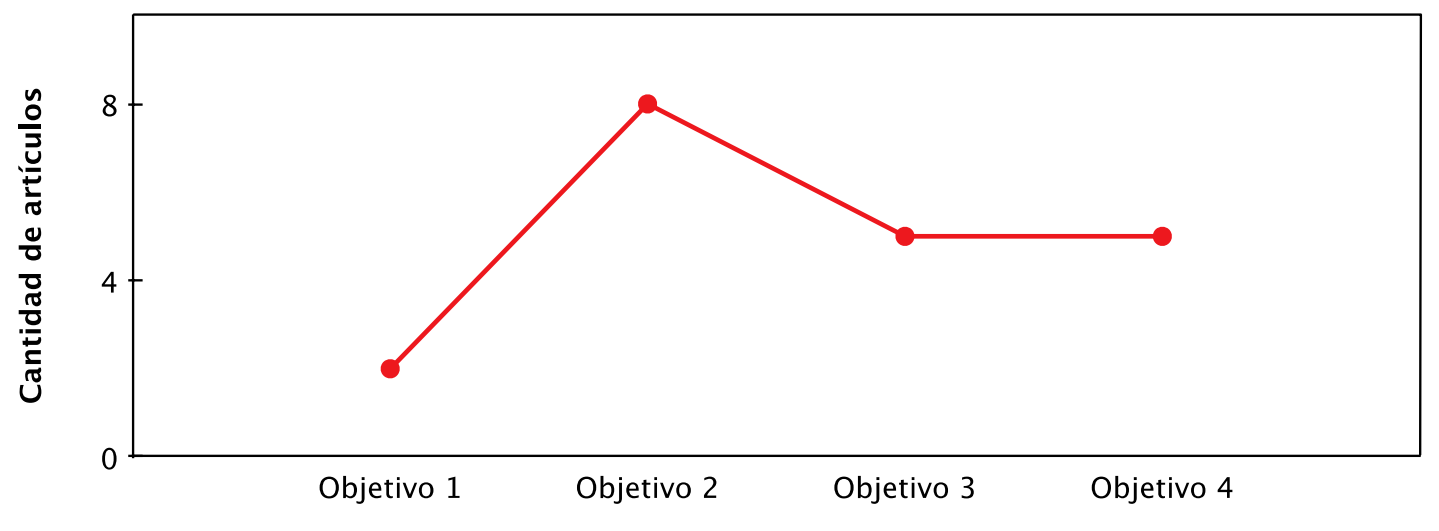

Objetivos de investigación

Fig. 6: Afinidad con los objetivos de investigación

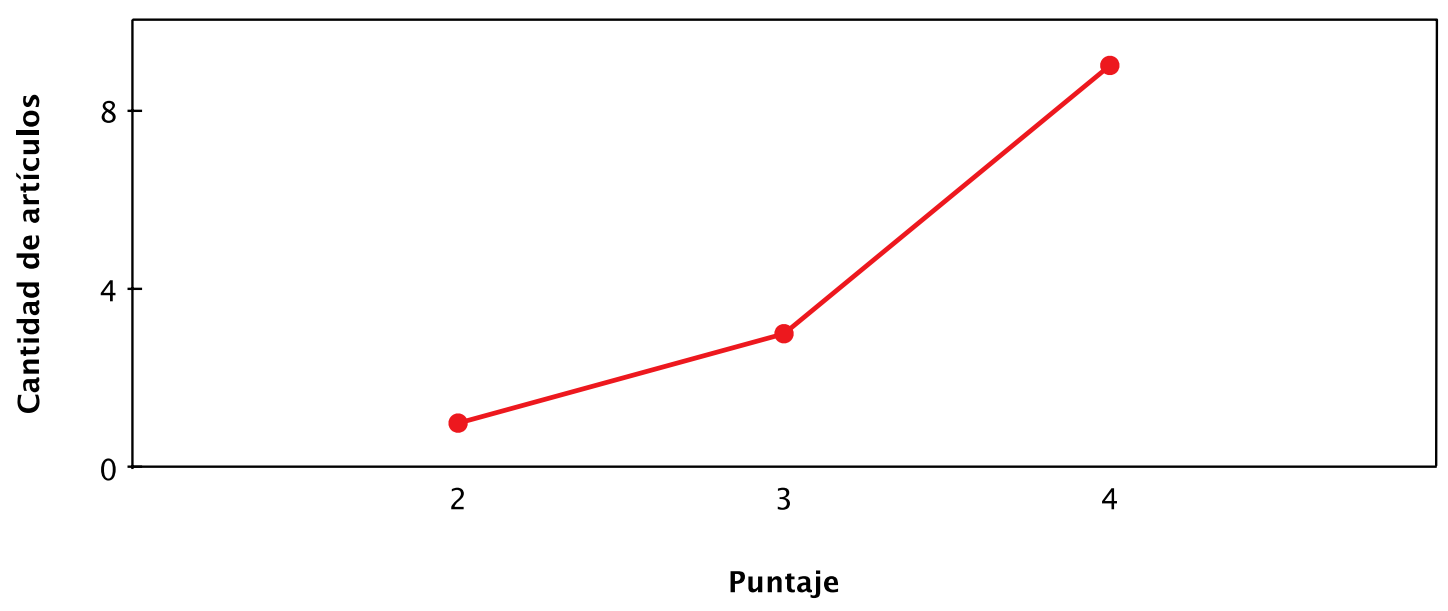

Fig. 7: Calificación de artículos (Escala de calificación de 1 a 5)

\section{Comparación de resultados obtenidos}

De los 13 artículos obtenidos durante la ejecución del proceso propuesto, 5 de ellos se encuentran en el estudio de RSL de Dias de Carvalho et al. (2014), estudio tomado como referencia. La diferencia que existe entre los artículos encontrados usando el proceso y la herramienta propuesta, y los encontrados por Dias de Carvalho et al., se debe particularmente a dos situaciones. La primera situación está relacionada con los problemas actuales de interoperación de los motores de búsqueda con Loxo, lo que limita el número de artículos a procesar; en el caso de SpringerLink se limita a 1000 la descarga de títulos, en el caso de ACM 
el motor se desconecta periódicamente de Loxo sin documentar errores tratables. La segunda situación, que resulta en artículos encontrados por Loxo y no encontrados por Dias de Carvalho et al., se relaciona con la capacidad del proceso y la herramienta Loxo de procesar sistemáticamente los artículos encontrados, evitando que se dejen por fuera del estudio títulos que deben ser considerados de acuerdo con los objetivos definidos de la investigación.

\section{CONCLUSIONES}

En este artículo se presenta un proceso de VT de publicaciones técnicas que integra la norma UNE 166006:2011 (2011) para la VT y el protocolo para realización de revisiones sistemáticas de literatura de Kitchenham y Brereton (2013). La norma UNE 166006:2011 enuncia los conceptos y las actividades que enmarcan el proceso de VT, sin embargo, las descripciones dadas por esta norma son de alto nivel y omiten detalles procedimentales de implementación. En el análisis de esta situación se evidencia que una organización para cumplir con sus motivadores de negocio debe contar con un proceso claro, organizado y sistemático para la VT que indique no solo como plantear la búsqueda sino también como procesar los resultados. Además, dicho proceso debe tener en cuenta la reducción del riesgo en la toma de decisiones y los tiempos de respuesta esperados por la estrategia que les permita cumplir con la competitividad. El objetivo con el proceso propuesto es complementar el proceso de la VT con elementos procedimentales de buenas prácticas para la búsqueda de publicaciones técnicas documentados en el protocolo de realización de RSL. Se presenta además una herramienta de soporte (Loxo) para facilitar la ejecución del proceso propuesto. Por medio de este proceso las organizaciones pueden lograr una planeación de la búsqueda de publicaciones técnicas más fluida donde el investigador puede dirigir sus esfuerzos en lo que quiere hacer y en su utilidad directa para las actividades posteriores. Para validar el proceso propuesto y la herramienta Loxo, éstos dos se aplicaron en el contexto de un proyecto de VT en una organización. La validación realizada permite conocer la asertividad del proceso y la herramienta y, además, los compara contra un proceso de RSL que no incluye principios de VT y que fue desarrollado manualmente. Tanto el proceso propuesto como la herramienta Loxo solo abarcan las actividades correspondientes a la búsqueda de publicaciones técnicas, las demás actividades de la VT requieren elementos procedimentales diferentes que no fueron abordados en este trabajo.

\section{REFERENCIAS}

Abdala, N. K., Revisión Sistemática de Literatura: Variabilidad de Atributos de Calidad en Líneas de Producto de Software, Tesis de Maestría, Facultad de Ingeniería, Universidad Icesi, Cali, Colombia (2014)

Bass, L., Clements, P., y Kazman, R., Software Architecture in Practice, $3^{\text {a }}$ edición, Addison-Wesley Professional (2012)

Biolchini, J.; Gomes Mian, P.; Cruz Natali, A. C. y Horta Travassos, G., Systematic Review in Software Engineering, Technical report RT-ES 679/05 (en línea;

http://www.cin.ufpe.br/ in1037/leitura/systematicReviewSE-COPPE.pdf, acceso: 6 de Noviembre 2015), Rio de Janeiro, Brasil (2005)

UNE 166006:2011, Norma Española sobre Gestión de la I+D+i: Sistemas de Vigilancia Tecnológica e Inteligencia Competitiva, Madrid, España (2011)

Dias de Carvalho, D.; Fernandes Chagas, L.; Magalhães Lima, A. y Lima Reis, C. A., Software Process Lines: A Systematic Literature Review, Software Process Improvement and Capability Determination, Vol. 477, pp. 118-130, Springer International Publishing, Vilnius, Lithuania (2014)

Dieste, O. y Padua, A. G., Developing Search Strategies for Detecting Relevant Experiments for Systematic Reviews, First International Symposium on Empirical Software Engineering and Measurement, IEEE, 215224, Madrid, España (2007)

Johannessen, J.-A. y Olsen, B., The future of value creation and innovations: Aspects of a theory of value creation and innovation in a global knowledge economy, International Journal of Information Management, 30(6), 502-511 (2010)

Kitchenham, B., Procedures for Performing Systematic Reviews, Joint Technical Report TR/SE-0401 / 0400011T.1 (en línea http://www.inf.ufsc.br/ awangenh/kitchenham.pdf, acceso: 6 de Noviembre 2015), Keele, UK, Sydney, Australia (2004)

Kitchenham, B. y Brereton, P., A systematic review of systematic review process research in software engineering, Information and Software Technology, 55(12), 2049-2075 (2013) 
Kitchenham, B. y Charters, S., Guidelines for performing Systematic Literature Reviews in Software Engineering, Technical Report EBSE-2007-01, Keele y Durham, UK (2007)

Kitchenham, B.; Sjøberg, D. I. K.; Brereton, O. P.; Budgen, D.; Dybå, T.; Höst, M.; Pfahl, D. y Runeson, P., Can we evaluate the quality of software engineering experiments?, ACM-IEEE International Symposium on Empirical Software Engineering and Measurement, ACM, 1-8, Bolzano-Bozen, Italy (2010)

López, A. C. y Mendez, D. F., Protocolo de vigilancia tecnológica a publicaciones técnicas, Tesis de Maestría, Facultad de Ingeniería, Universidad Icesi, Cali, Colombia (2015)

Marshall, C. y Brereton, P., Tools to support systematic literature reviews in software engineering: $A$ mapping study, International Symposium on Empirical Software Engineering and Measurement, IEEE, 296299, Baltimore, MD, USA (2013)

Petersen, K.; Feldt, R.; Mujtaba, S. y Mattsson, M., Systematic mapping studies in software engineering, 12th International Conference on Evaluation and Assessment in Software Engineering, British Computer Society, 68-77, Swinton, UK (2008).

Skoglund, M. y Runeson, P., Reference-based search strategies in systematic reviews, 13th International Conference on Evaluation and Assessment in Software Engineering, British Computer Society, 31-40. Swinton, UK (2009)

Staples, M. y Niazi, M., Experiences using systematic review guidelines, Journal of Systems and Software, 80(9), 1425-1437 (2007)

Yang, Z.; Li, Z.; Jin, Z. y Chen, Y., A Systematic Literature Review of Requirements Modeling and Analysis for Self-adaptive Systems, in Requirements Engineering: Foundation for Software Quality, pp. 55-71, Springer International Publishing, Essen, Germany (2014) 